\title{
RDUS
}

Revue de DROIT

UNIVERSITÉ DE SHERBROOKE

Titre : $\quad$ L'ALLOCATION DES RESSOURCES ET SON IMPACT SUR LA GESTION DES RESSOURCES HUMAINES DANS LES CENTRES HOSPITALIERS

Auteur(s): $\quad$ Léandre NADEAU

Revue: $\quad$ RDUS, 1989-1990, volume 20, numéro 2

Pages: $\quad 265-283$

ISSN : $\quad 0317-9656$

Éditeur : $\quad$ Université de Sherbrooke. Faculté de droit.

URI : $\quad$ http://hdl.handle.net/11143/13556

DOI : https://doi.org/10.17118/11143/13556 
Page vide laissée intentionnellement. 


\section{L'ALLOCATION DES RESSOURCES ET SON IMPACT SUR LA GESTION DES RESSOURCES HUMAINES DANS LES CENTRES HOSPITALIERS}

par Léandre NADEAU*

* Vice-président exécutif, Association des hôpitaux du Québec. 
SOMMAIRE

LA SITUATION DES FINANCES PUBLIQUES . . . . . . 267

LA SITUATION FINANCIERE DES CENTRES HOSPITA-

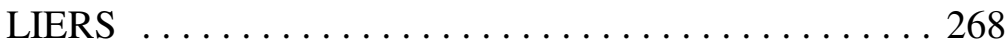

LA MARGE DE MANOEUVRE DE L'ETABLISSEMENT

EST EXTREMEMENT RESTREINTE . . . . . . . . . . . . 269

LA SITUATION DES RESSOURCES HUMAINES . . . . . . 270

CONCLUSION ....................... 275 
Au cours de la décennie 1980, la gestion des ressources humaines dans le secteur public, et en particulier dans le réseau hospitalier, a été considérablement influencée par les orientations de la politique financière du gouvernement. Je vous entretiendrai donc en premier lieu de la politique financière du gouvernement. Par la suite, je dégagerai les impacts de cette politique sur la gestion des ressources humaines en milieu hospitalier.

\section{LA SITUATION DES FINANCES PUBLIQUES}

Depuis le début des années 1980, l'ensemble du secteur public et parapublic québécois a évolué dans un contexte budgétaire relativement restrictif. Cette situation n'est pas particulière au Québec puisque la plupart des pays industrialisés ont été contraints d'adopter une telle approche suite à la crise économique de 1982 et au développement fulgurant des capacités de production dans les pays asiatiques. Cette orientation était même antérieure à la crise économique de 1982 puisque dès la fin des années 1970, les pays formant le «groupe des sept» avaient convenu de limiter la croissance des dépenses publiques à un taux se situant en deça de la croissance de la production nationale. Ces pays étaient d'avis qu'une telle orientation était nécessaire pour corriger les déficits budgétaires, atténuer l'inflation et rétablir à moyen terme leurs capacités concurrentielles.

Il est maintenant évident que le régime d'amaigrissement en vigueur depuis bientôt 10 ans a eu des impacts importants dans à peu près tous les secteurs de dépenses publiques. Globalement, les dépenses du gouvernement du Québec ont été réduites en pourcentage du produit intérieur brut (PIB) de 25,7\% à 21,4\% de 1983-1984 à 1989-1990. Au total, au cours des quatre dernières années, c'est de quelque 5,9 milliards de dollars que le gouvernement du Québec a freiné l'évolution des dépenses gouvernementales, en limitant leur croissance à un taux le plus près possible du taux d'inflation. Toutes les missions gouvernementales ont dû, en quelque sorte, contribuer à cet effort d'assainissement des finances publiques.

Ainsi, la mission économique a vu ses dépenses en pourcentage du PIB diminuer de 0,3\% (soit $391 \mathrm{M} \$$ ), la mission éducative et culturelle de $1,1 \%$ (soit $1654 \mathrm{M} \$$ ), la mission sociale de $1,2 \%$ (soit 1 $927 \mathrm{M}$ \$) et la mission administrative de $0,8 \%$ (soit $1189 \mathrm{M}$ \$). Les dépenses du secteur santé et adaptation sociale, en pourcentage du PIB, ont été réduites de 0,5\% (soit 768 M \$). (Annexe 1) 
Dans l'esprit du gouvernement, ce régime était absolument nécessaire pour s'attaquer à trois problèmes majeurs. En premier lieu, il a cherché à réduire le fardeau fiscal des contribuables afin de le rendre plus concurrentiel avec, notamment, l'Ontario. Deuxièmement, il a tenté de ramener le déficit au niveau des dépenses de capital afin de ne pas être obligé d'emprunter pour les dépenses courantes et ainsi hypothéquer les générations futures. Enfin, il a dû faire face à la diminution constante des transferts fédéraux. La réduction des dépenses publiques pour un montant de 5,9 milliards de dollars a servi à ces fins. (Annexe 2)

\section{LA SITUATION FINANCIERE DES CENTRES HOSPITALIERS}

En somme, compte tenu de ces objectifs, il restait très peu de disponibilités financières pour répondre à la croissance de la demande de services résultant du vieillissement de la population et du développement de la technologie médicale.

Durant la première moitié des années 1980, la situation financière du réseau hospitalier s'est détériorée très rapidement: le déficit des établissements en difficulté passa de 29,5 M \$ en 1983-1984 à 78,3 M \$ en 1984-1985 et à 160,5 M \$ en 1985-1986.

Depuis 1986, la situation financière des centres hospitaliers est beaucoup plus saine. Elle est le résultat d'un cadre de financement plus réaliste, de règles ministérielles beaucoup plus contraignantes et d'efforts systématiques des gestionnaires du réseau hospitalier et du Ministère visant l'atteinte de l'équilibre budgétaire. Malgré tout, on observe dans un certain nombre d'hôpitaux des déficits annuels représentant près de pour cent du budget des établissements, dont une bonne part s'explique par l'arrivée de médecins spécialistes dans les hôpitaux spécialisés des régions intermédiaires ou éloignées.

Le resserrement du cadre de gestion a cependant eu des impacts sur l'accessibilité aux services. L'Association des hôpitaux du Québec a calculé que les centres hospitaliers ont été contraints de réduire, de 1981 à 1987, d'environ 7,3 \% l'offre de services de soins de courte durée pour les bénéficiaires de moins de soixante-cinq ans, soit 1,25\% par année. (Annexe 3) Le nombre de lits réellement utilisés dans les hôpitaux de courte durée a été réduit à chaque année. De plus, les centres hospitaliers accumulent des retards dans le développement de plusieurs services. 
Par ailleurs, le contexte serré des finances publiques des dix dernières années a également eu des impacts très importants sur la gestion des ressources humaines.

\section{LA MARGE DE MANOEUVRE DE L'ETABLISSEMENT EST EXTREMEMENT RESTREINTE}

Examinons maintenant le problème d'allocation du point de vue de l'établissement.

Chaque centre hospitalier reçoit annuellement du gouvernement un budget global lui permettant d'assumer les dépenses nécessaires à l'accomplissement de sa mission.

En théorie, il peut affecter ses ressources financières comme il le juge nécessaire pour satisfaire la demande de services. Cependant, dans les faits, sa marge de manoeuvre est très limitée. Elle est réduite par des contraintes de toute sorte. Les plus importantes sont les suivantes:

la demande de soins s'accroît d'année en année et à cet égard, le vieillissement de la population du Québec apporte une pression très forte sur le budget des établissements;

les conventions collectives sont très élaborées, elles varient entre 200 et 485 pages.

- $\quad$ comme toute entreprise du Québec, les centres hospitaliers doivent s'adapter et respecter les lois et règlements fixés par nos gouvernements. L'exemple le plus récent est le système d'identification des matières dangereuses utilisées au travail (SIMDUT). Dans un établissement universitaire ultra-spécialisé, on dénombre environ 3000 produits différents dont environ $30 \%$ sont réglementés par le SIMDUT et pour lesquels on devra informer et former tous les utilisateurs par des sessions variant d'une heure à un jour. Ce travail est essentiel. Cependant, comme nos centres hospitaliers fonctionnent avec un budget fermé, ils devront probablement assumer cette responsabilité à même leur budget global sans avoir la possibilité de refiler la facture au consommateur comme pourra le faire toute autre entreprise.

par ailleurs, plusieurs changements dans l'organisation du centre hospitalier, telles une réorganisation administrative, une abolition de poste, une fermeture de service ou l'ouverture d'un 
nouveau service, doivent être précédés d'une autorisation préalable soit du Ministère soit du Conseil régional.

Ces contraintes réduisent la marge de manoeuvre et la capacité de réagir rapidement aux problèmes rencontrés.

\section{LA SITUATION DES RESSOURCES HUMAINES}

Comme vous le savez, la répartition de la dépense dans un centre hospitalier de courte durée est d'environ $20 \%$ en fourniture et $80 \%$ en salaire.

Les fournitures comprennent principalement des dépenses incompressibles qui, souvent même, s'accroissent à un rythme beaucoup plus rapide que le taux d'accroissement du budget attribué par le Ministère. Ce sont, entre autres, les produits chirurgicaux et pharmaceutiques, les prothèses et orthèses, l'alimentation, l'électricité et le chauffage.

En conséquence, les impacts du cadre budgétaire étroit des années 80 ont pour une très grande part été assumés par un contrôle très serré des heures travaillées. Cette contraction du volume de la main-d'oeuvre a occasionné, entre autres, une réduction dans la composition des équipes de travail.

\section{A. DES EQUIPES DE TRAVAIL MINIMALES}

Prenons l'exemple de la périnatalité et de la pouponnière.

Dans ces secteurs, les équipes de travail dans les années 70 étaient généralement construites de façon à pouvoir répondre à la charge moyenne de travail ou, dans le jargon du milieu, à un volume de travail établi selon le taux moyen d'occupation des lits. Les restrictions budgétaires ont forcé les établissements à réévaluer ces équipes et à les ajuster à la baisse pour ne répondre qu'au taux minimum d'occupation.

En pratique, dans le passé, lorsque la charge de travail était inférieure à la moyenne, nous ne retournions pas le personnel chez lui: plus de temps était donc disponible pour accomplir les tâches requises.

Aujourd'hui, l'équipe étant réduite au minimum, on ajuste à la hausse le nombre de personnes présentes si la charge s'accroît mais cela nécessite une révision quasi-quotidienne et l'équipe ne bénéficie plus de ce que nous pourrions appeler des périodes de répit. 
Dans les autres secteurs de l'hôpital, les équipes de travail ont été réévaluées et toutes les heures jugées superflues ont été défalquées.

En conséquence, même si certaines techniques de travail furent simplifiées ou que certaines activités furent carrément abandonnées, tous les travailleurs et travailleuses durent accepter un accroissement de leur charge de travail.

\section{B. PRECARITE DES EMPLOIS}

Cette situation eut comme effet de faire surgir un certain nombre d'emplois précaires.

Le nombre de postes à temps partiel, qui était déjà élevé depuis l'attribution par la convention collective d'une fin de semaine sur deux à tout salarié s'accrût sensiblement. La proportion des heures travaillées par les salariés à temps partiel a augmenté beaucoup entre 1980-1981 et 1986-1987, passant de 26,7 \% à 35,0\%. (Annexe 4) Mais ce qui est particulièrement préoccupant, c'est le nombre d'heures effectuées par les salariés temporaires (ou les occasionnels) qui demeure élevé, soit 15,3\% des heures travaillées dans le réseau. C'est surtout cette catégorie de personnes qui subit la précarité de l'emploi.

Nous admettons qu'il y a beaucoup d'employés à temps partiel. Autant du côté patronal que syndical, les intervenants s'accordent sur la nécessité d'augmenter le ratio de postes à temps complet. Mais les employés ne souhaitent pas tous et toutes travailler à temps complet.

Bien au contraire, un sondage réalisé en 1988, pour le compte du Comité patronal de négociation du secteur de la santé et des services sociaux, démontre que:

- $\quad 71 \%$ des salariés à temps partiel travaillent à temps partiel par choix personnel. Il faut cependant nuancer entre temps partiel régulier et temporaire;

- $\quad 74 \%$ des salariés à temps partiel régulier sont satisfaits de leur statut; cependant, les employés à temps partiel temporaire le sont beaucoup moins; seulement $42 \%$ des salariés à temps partiel temporaire sont satisfaits de leur statut.

Pour l'ensemble de tous les employés, s'ils avaient le choix:

- $\quad 56 \%$ opteraient pour le statut de temps complet;

- $\quad 40 \%$ opteraient pour le statut de temps partiel; 
- $\quad 4 \%$ opteraient pour effectuer du remplacement.

Les efforts des établissements consisteront donc au cours des prochains mois, et avec la collaboration des syndicats locaux, à entreprendre une démarche visant à convertir en heures régulières et en emplois permanents les heures de remplacement qui peuvent être converties.

\section{LA CHARGE DE TRAVAIL}

Les restrictions budgétaires et la réorganisation des équipes de travail qui ont suivi, couplées à l'augmentation des demandes de la clientèle et à l'alourdissement des bénéficiaires, ont sans contredit augmenté la charge de travail de l'ensemble du personnel.

Pour étayer cet avancé, on remarque à l'annexe 5 concernant l'évolution des heures travaillées de la main-d'oeuvre hospitalière de 1984 à 1989, que globalement la productivité s'est accrue de 3,81\%.

Cet accroissement de la charge de travail survient alors même que la clientèle s'alourdit et qu'elle requiert des soins de plus en plus complexes.

En effet, le vieillissement des patients hospitalisés, les critères d'admission plus sélectifs et l'utilisation accrue de structures de soins plus légères se combinent pour «alourdir» chacun des cas traités en hospitalisation. Certaines données individuelles recueillies par des systèmes de mesure de la charge des besoins en soins infirmiers confirment ce phénomène non seulement dans les centres hospitaliers de soins de courte durée mais aussi dans ceux de soins de longue durée.

(Annexe 6)

L'alourdissement des cas traités en hospitalisation est aussi associé à une complexification de plus en plus grande du profil diagnostique. Les tumeurs, les maladies de l'appareil circulatoire, les lésions traumatiques et empoisonnements et les maladies du système nerveux et des organes des sens sont les catégories diagnostiques qui voient le plus leur part relative augmenter entre 1981-82 et 1986-87.

Ces diagnostics sont aussi caractérisés par des séjours moyens supérieurs à la moyenne et par une utilisation plus intense de ressources diagnostiques et thérapeutiques spécialisées. Dans les cas de tumeurs malignes, des cardiopathies, des fractures, des maladies de l'oeil et de ses annexes, ces tendances à une plus forte incidence se 
manifestent, même après avoir tenu compte du vieillissement de la population.

Faute de données québécoises sur un indice de complexité des cas, il est intéressant de citer l'expérience hospitalière ontarienne qui montre que la valeur d'un tel indice, ramenée à un cas moyen, a crû de 0,3\% par année entre 1984-85 et 1986-87.

Cette situation est fortement ressentie par tout le personnel mais plus particulièrement par le personnel directement affecté aux soins des bénéficiaires dont les techniciens et professionnels de la santé. Le temps disponible pour le traitement de chaque bénéficiaire étant diminué, ils ne peuvent accorder à celui-ci toute l'attention à laquelle il a droit et il en résulte un sentiment de dévalorisation.

Nous comprenons cette situation et estimons que si le réseau ne dispose pas au cours des prochaines années de ressources additionnelles suffisantes, le phénomène de l'alourdissement des cas traités remettra même en question le volume de patients actuellement traités.

\section{INCIDENCE DES CONVENTIONS COLLECTIVES EN PERIODE DE RESTRICTION BUDGETAIRE}

En période de restriction budgétaire, la gestion des conventions collectives, dont les dispositions peuvent s'étaler sur plusieurs centaines de pages, représente une course à obstacle extrêmement complexe.

Nous avons identifié que dans notre réseau, il existe plus de 250 titres d'emploi différents. Plusieurs libellés de ces titres d'emploi sont construits de façon telle que la tâche faite par une catégorie de salariés ne peut être accomplie par quelqu'un d'un autre titre d'emploi. Encore ici, lorsque les ressources sont abondantes, on réalise peu l'impact de cette situation sur les services. Mais en période de pénurie, alors qu'on doit revoir la composition des équipes de travail et obtenir plus de polyvalence du personnel, les titres d'emploi, construits de façon mutuellement exclusive, ressortent avec leurs conséquences: soit que la situation est maintenue même si elle est inefficace, soit que deux postes à temps partiel sont créés ou qu'une fusion de poste est acceptée par le syndicat. A noter qu'un refus du syndicat, à cet égard, oblige l'employeur au statu quo ante.

Comme nous en avons fait état précédemment, lorsque les équipes de travail sont établies pour répondre au volume maximum 
d'activités ou même au volume normal d'activités, il est facile et parfois même économique d'accorder des congés en période de ralentissement d'activités, tout comme il est plus simple d'assumer l'entraînement du nouveau personnel.

Cependant, lorsque les équipes de travail sont au minimum et que leur charge de travail est très grande, toute absence est dramatique et l'entraînement du nouveau personnel est toujours perçu comme un fardeau pour le personnel en place. On exige du remplaçant qu'il soit en mesure de s'intégrer immédiatement et pleinement à l'équipe.

La même problématique survient lors des attributions de postes et des remplacements. Les équipes de travail tolèrent difficilement, en période de pénurie, qu'une personne prenne plusieurs jours à apprendre ce que son prédécesseur faisait seul sans l'aide et sans la surveillance des autres.

La charge de travail étant forte, l'inefficacité au sein de l'équipe n'est pas tolérée car elle se traduit par une surcharge de travail pour les autres.

Il est utile d'avoir une convention collective pour clarifier les responsabilités et les droits et développer et consolider la confiance entre les parties. Mais au moment où nous gérons la décroissance et lorsque les ressources sont limitées, cette convention collective devrait permettre une affectation efficiente et efficace des ressources humaines et financières.

\section{E. LES TECHNICIENS ET PROFESSIONNELS DE LA SANTE}

Les impacts dont nous venons de parler se vivent souvent avec plus d'acuité par les techniciens et les professionnels de la santé.

Comme nous le disions précédemment, ces personnes travaillant directement auprès des bénéficiaires, elles ressentent plus encore le sentiment de dévalorisation causé par l'accroissement du volume d'activités. Elles ont à traiter des cas plus complexes et en plus grand nombre qu'avant.

Elles doivent posséder une expertise plus grande, s'adapter plus rapidement aux nouvelles situations et travailler avec des technologies de plus en plus complexes qui évoluent à un rythme quasi infernal.

D'autre part, il existe parmi ce groupe de personnes une pénurie de main-d'oeuvre encore plus accentuée dans certaines régions de la 
province. La situation des infirmières, bien que la plus connue, n'est pas la seule. A titre d'exemple: la main-d'oeuvre disponible est inférieure à la demande chez les physiothérapeutes, les ergothérapeutes, les techniciens en radio-oncologie et les techniciens en médecine nucléaire.

Nous nous retrouvons alors avec des postes vacants et des remplacements non comblés qui conduisent à un accroissement supplémentaire de la charge de travail et l'obligation morale pour plusieurs d'effectuer très régulièrement des heures supplémentaires.

\section{F. LES CADRES}

Dans cette problématique on oublie souvent les cadres. Pourtant, ils vivent pratiquement les mêmes problèmes que les salariés. Ils ont aussi subi une diminution d'effectifs, soit de 4,3\% entre 1980-1981 et 1986-1987; des postes ont été fusionnés, des tâches ont été regroupées et il n'est pas rare dans le réseau de rencontrer un gestionnaire supervisant plus de 45 salariés dans son service. Le suivi budgétaire étant plus serré, on leur demande de rendre compte plus régulièrement. L'ajustement des équipes de travail nécessite une évaluation parfois quotidienne.

Ils reçoivent à la fois les plaintes des salariés et des clients face à des décisions qui leurs sont imposées par des contraintes externes. Ils agissent comme tampon entre la clientèle, le personnel et la haute direction.

Les cadres doivent également s'adapter aux changements rapides de leur clientèle, à la modification des soins et services qu'ils ont à dispenser ainsi qu'à la technologie mise à la disposition de leur équipe de travail.

\section{CONCLUSION}

Notre conclusion portera sur deux plans et fera état des perspectives en gestion des ressources humaines et des perspectives financières.

L'Association des hôpitaux du Québec est consciente qu'un virage est nécessaire sur ces deux plans.

Dans le domaine de la gestion des ressources humaines le virage est déjà entrepris. Depuis plus de cinq ans, nous supportons les 
établissements et les incitons à modifier leur philosophie de gestion afin que l'on cesse de limiter le débat simplement au niveau de la négociation de la convention collective, de l'application de cette convention et du traitement des griefs.

La gestion des ressources humaines doit tenir compte à la fois des aspirations des employés et de la nécessité d'offrir des services de qualité.

C'est dans cette optique qu'en 1988 nous avons lancé sur la place publique le vaste débat relié à la profession d'infirmière.

C'est aussi sur cette base que nos objectifs de négociation dans la présente ronde furent déterminés.

Nous souhaitons accroître la stabilité de la main-d'oeuvre notamment en augmentant le nombre de postes permanents.

Nous voulons revaloriser la présence au travail et responsabiliser les parties en terme de droits et obligations, tendre vers une déréglementation des conventions collectives et une appropriation par les instances locales des modalités d'application des conditions de travail.

La FIIQ et la FTQ ont compris le bien-fondé de nos objectifs et ont accepté des modifications aux conventions collectives en conséquence. C'est ainsi, à titre d'exemple, que nous avons obtenu pour les salariés à temps partiel une priorité de remplacement dans leur service et nous nous sommes engagés à effectuer, dans chaque centre hospitalier, une démarche de conversion des heures de remplacement en postes.

Il appartiendra aux établissements de procéder localement. Soyez assurés cependant que l'AHQ apportera à ses membres toute la disponibilité et le support nécessaires afin que les résultats soient satisfaisants.

En ce qui concerne les perspectives financières des centres hospitaliers, il apparaît clairement qu'un effort supplémentaire de financement devra être fait au cours des prochaines années pour faciliter la gestion des ressources humaines et maintenir l'accessibilité aux services, sans pour autant détériorer la situation financière des établissements. 
Le redressement de l'état des finances publiques du Québec, réalisé par le gouvernement au cours des dernières années, nous permet d'être optimistes quant à sa capacité, à moyen terme, de financer adéquatement l'évolution du réseau hospitalier. Cependant, la conjoncture actuelle d'un ralentissement économique et d'un important déficit fédéral font craindre que les disponibilités financières du gouvernement demeureront extrêmement limitées à court terme.

Dans ces conditions, l'Association des hôpitaux du Québec croit qu'il est temps de s'interroger très sérieusement sur l'opportunité de diversifier les sources de financement du réseau. De plus, l'Association est d'avis que le gouvernement fédéral ne pourra pas continuer de réduire son financement des programmes de santé tout en maintenant des normes extrêmement rigides sur la gratuité des services. 
ANNEXE 1 
ANNEXE 2 
ANNEXE 3 
ANNEXE 4 
(1990) 20 R.D.U.S.

ANNEXE 5 
ANNEXE 6 